\title{
Apply hyperanalytic shearlet transform to geometric separation
}

\author{
Shuaiqi Liu ${ }^{1,2^{*}}$, Shaohai Hu${ }^{1 \dagger}$, Mingzhu Shi², Zhong Zhang ${ }^{2}$ and Shuang Liu²
}

\begin{abstract}
This paper first proposes a novel image separation method based on the hyperanalytic shearlet. By combining the advantages of both the hyperanalytic wavelet transform and the shear operation, hyperanalytic shearlet is easy to implement and also has a low redundancy. By using such transform and the orthonormal wavelet, a new geometric separation dictionary is obtained which can sparsely represent points and curviline singularities, respectively. In order to get the different components of image faster and more accurate, a fast alternating direction method (FADM) is used to train the dictionary. Our algorithm can greatly improve the computational efficiency without causing damage to the accuracy of image separation. Furthermore, a proper measure to evaluate the separation performance called sep-degree is defined. The experimental results have demonstrated the proposed method's effectiveness and superiority.
\end{abstract}

Keywords: Hyperanalytic shearlet; Geometric separation; Sparse approximation; Separation degree

\section{Introduction}

Astronomical images of the galaxy can be modeled as a superposition of pointlike and curvelike structures. In the further analysis, astronomers typically face the problem of extracting the stars from filaments which mostly are trajectory of the particle and hence separating pointlike from curvelike structures. Thus, this area is greatly attracting scholars' attention in order to find efficient methodologies for accurately conquering this task.

Although this problem seems unsolvable - the problem is underdetermined, as there are two unknown (the images should be extracted) and only one known data (the given image) - experimental results using morphological component analysis (MAC) [1,2] suggest that such a problem is possibly solvable when we get the prior information about the type of decomposed features and enough morphological difference between those features. For the separation of pointlike and curvelike features, perfect results have been achieved by employing a dictionary consisting

\footnotetext{
*Correspondence: shdkj-1918@163.com

${ }^{\dagger}$ Equal contributors

1 Institute of Information Science, Beijing Jiaotong University, Shangyuancun 3, Haidian district, Beijing, 100044 China

${ }^{2}$ College of Electronic and Communication Engineering, Tianjin Normal University, Binshuixi Road 339, Xiqing district, Tianjin 300387, China
}

of wavelet and curvelet with combination with $l_{1}$ minimization techniques [2]. We know that pointlike structures can be optimally sparse representation in wavelet dictionary, and curvelike structures also can be optimally sparse representation in curvelet dictionary. Thus, the pointlike structures can be expressed by wavelet and the curvelike structures can be expressed by curvelet with applying $l_{1}$ minimization to the expansion coefficients, and then the image can be separated automatically.

The current papers $[3,4]$ give a newly combined dictionary of orthonormal wavelet and shearlet for separating pointlike and curvelike features. And numerical results give evidence that the shearlet-based decomposition algorithms have a superior behavior than curveletbased algorithms in [4]. In [3,4], the authors introduce a nonsubsampled shearlet transform to design a separation dictionary which greatly increase the redundancy. And they choose an old method called block coordinate relaxation (BCR) to solve the $l_{1}$ problem which is not only time-consuming but also unable to get an accurate result. Thus, in this paper, a novel approach to the separation of pointlike and curvelike features based on hyperanalytic shearlet is proposed.

Shearlet transform is a new multi-scale geometric analysis algorithm which inherits the advantages of the contourlet and curvelet transforms. It is also an optimal

\section{是 Springer}

(c) 2014 Liu et al.: licensee Springer. This is an Open Access article distributed under the terms of the Creative Commons

Attribution License (http://creativecommons.org/licenses/by/4.0), which permits unrestricted use, distribution, and reproduction in any medium, provided the original work is properly credited. 
approximation presentation [5,6] for singular curve or surface that contains $C^{2}$ high-dimensional signals. A shift invariant shearlet is achieved by nonsubsampled Laplace pyramid in [6]. This construction not only greatly increases the redundancy of the transform but also slows down the calculation sharply. From [7], we can know that hyperanalytic wavelet (HWT) just has limited redundancy to achieve multi-scale decomposition without shift sensitivity, which is similar to dual tree complex wavelet (DTCWT), but differently, this method is more easily achievable than dual tree complex wavelet. And the construction of hyperanalytic wavelet also greatly accelerates the computation. So we propose a new fast discrete shearlet called hyperanalytic shearlet transform. It is implemented by using hyperanalytic wavelet $[3,4]$ to achieve multi-scale decomposition, and then shear filter bank is applied to the high-pass coefficients. This new algorithm holds the advantages of simpler structure and higher sparsity. The hyperanalytic shearlet addresses the problem of the aliasing phenomenon and greatly reduces the redundancy and computing time compared to nonsubsampled shearlet transform. In our paper, we apply it to separate an image into its morphologically different contents. In order to get the different components of image faster and more accurate, we also use fast alternating direction method (FADM) instead of BCR to train dictionary. Our algorithm can greatly improve the computational efficiency without damage to the accuracy of image separation. To compare the performance difference between this new algorithm and the method in [4], we define a new measure of the separation called sep-degree. The experiment results will demonstrate that our scheme has a better separation effect.

This paper is organized as follows. Section 2 introduces the construction of hyperanalytic shearlet transform. Section 3 describes the mathematical theory of geometric separation of pointlike and curvelike features and applies a novel algorithm to separate an image into morphologically different contents. Section 4 illustrates the numerical results. Finally, Section 5 presents some conclusions.

\section{Hyperanalytic shearlet transform}

Shearlet transform theory is based on composite wavelet. In dimension $n=2$, the affine systems with composite dilations are the collections of the form:

$\mathcal{A}_{A B}(\psi)=\left\{\psi_{j, l, \boldsymbol{k}}(\boldsymbol{x})=|\operatorname{det} \boldsymbol{A}|^{j / 2} \psi\left(\boldsymbol{B}^{l} \boldsymbol{A}^{j} \boldsymbol{x}-\boldsymbol{k}\right): j, l \in \mathbb{Z}, \boldsymbol{k} \in \mathbb{Z}^{2}\right\}$

where $\psi \in L^{2}\left(\mathbb{R}^{2}\right), \boldsymbol{A}, \boldsymbol{B}$ are $2 \times 2$ invertible matrices and $|\operatorname{det} \boldsymbol{B}|=1$. For any $f \in \mathbb{R}^{2}, \mathcal{A}_{\boldsymbol{A} \boldsymbol{B}}(\psi)$ forms a Parseval frame (also called tight frame); we call the elements of $\mathcal{A}_{A B}(\psi)$ composite wavelet. Let $\boldsymbol{A}=\boldsymbol{A}_{0}=\left(\begin{array}{ll}4 & 0 \\ 0 & 2\end{array}\right)$ be the anisotropic dilation matrix, and $\boldsymbol{B}=\boldsymbol{B}_{0}=\left(\begin{array}{ll}1 & 1 \\ 0 & 1\end{array}\right)$ be shear matrix in (1); we can construct a tiling of the frequency as shown in Figure 1.

For any $\xi=\left(\xi_{1}, \xi_{2}\right) \in \hat{\mathbb{R}}^{2}, \xi_{1} \neq 0$, let $\psi^{(0)}(\xi)$ be given by the frequency, that is

$$
\hat{\psi}^{(0)}(\xi)=\hat{\psi}^{(0)}\left(\xi_{1}, \xi_{2}\right)=\hat{\psi}_{1}\left(\xi_{1}\right) \hat{\psi}_{2}\left(\frac{\xi_{2}}{\xi_{1}}\right)
$$

where $\hat{\psi}_{1}, \hat{\psi}_{2} \in C^{\infty}(\hat{\mathbb{R}}), \operatorname{supp} \hat{\psi}_{1} \subset\left[-\frac{1}{2},-\frac{1}{16}\right] \cup\left[\frac{1}{16}, \frac{1}{2}\right]$, $\operatorname{supp} \hat{\psi}_{2} \subset[-1,1]$. Then, you know $\operatorname{supp} \hat{\psi}^{(0)} \subset\left[-\frac{1}{2}, \frac{1}{2}\right]^{2}$.

From the above analysis, we can get the support range of the function $\psi_{j, l, k}$ in the frequency domain:

$$
\operatorname{supp} \hat{\psi}_{j, l, k}^{(0)} \subset\left\{\begin{array}{l}
\left(\xi_{1}, \xi_{2}\right): \xi_{1} \in\left[-2^{2 j-1},-2^{2 j-4}\right] \cup \\
{\left[2^{2 j-4}, 2^{2 j-1}\right],\left|\frac{\xi_{2}}{\xi_{1}}+l 2^{-j}\right| \leq 2^{-j}}
\end{array}\right\}
$$

That is, each element $\hat{\psi_{j, l, k}}$ is supported on a pair of trapezoids, of approximate size $2^{2 j} \times 2^{j}$, oriented along lines of slope $l 2^{-j}$ (see Figure 2).

Let $D_{0}=\left\{\left(\xi_{1}, \xi_{2}\right) \in \hat{\mathbb{R}}^{2}:\left|\xi_{1}\right| \geq \frac{1}{8},\left|\frac{\xi_{1}}{\xi_{2}}\right| \leq 1\right\}$, that means $\forall\left(\xi_{1}, \xi_{2}\right) \in D_{0}$, the function group $\left\{\hat{\psi}^{(0)}\left(\boldsymbol{\xi} \boldsymbol{A}_{0}^{-j} \boldsymbol{B}_{0}^{-l}\right)\right\}$ forms a tiling of $D_{0}$ as shown in Figure 1 , notes that $D_{0}$ is illustrated in solid line.

From [6], we know that the following set is a Parseval frame for $L^{2}\left(D_{0}\right)$.

$$
\left\{\psi_{j, l, \boldsymbol{k}}^{(0)}(\boldsymbol{x})=2^{\frac{3 j}{2}} \psi^{(0)}\left(\boldsymbol{B}_{0}^{l} \boldsymbol{A}_{0}^{j} \boldsymbol{x}-\boldsymbol{k}\right): j \geq 0,-2^{j} \leq l \leq 2^{j}-1, \boldsymbol{k} \in \mathbb{Z}^{2}\right\}
$$

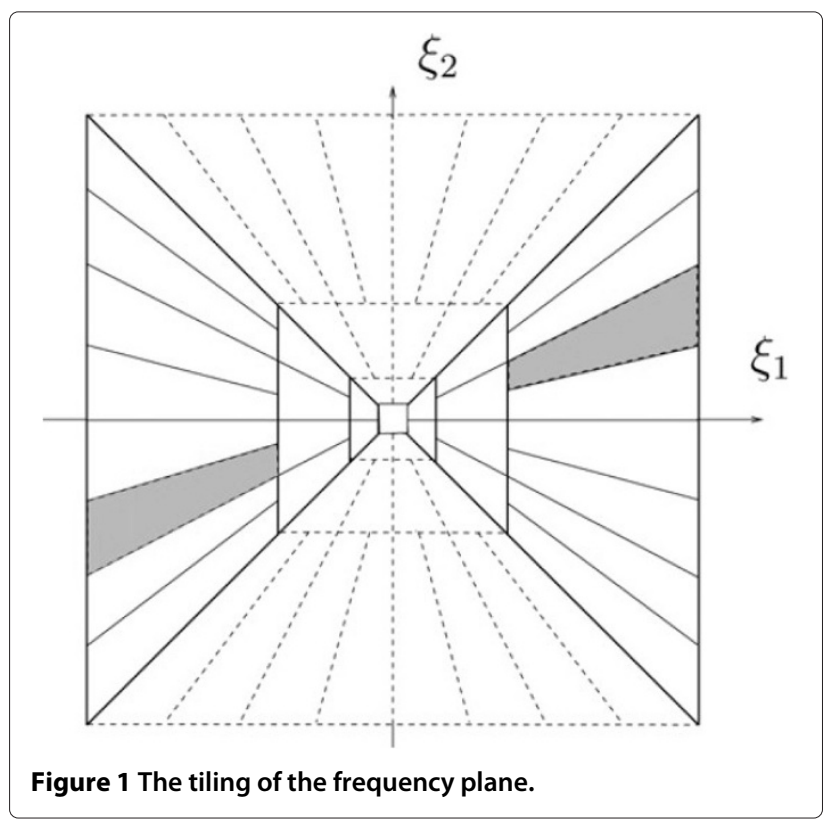




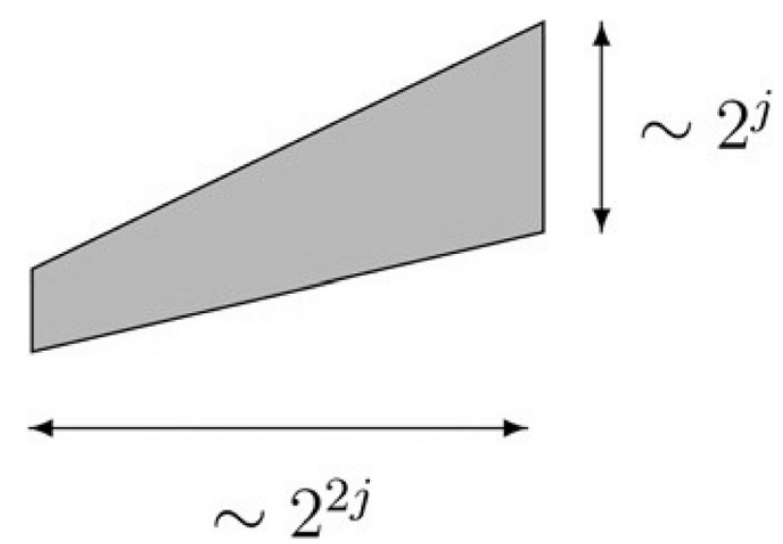

Figure 2 The frequency support of $\psi_{j, l, k}$.

Similarly, we can construct the other half tiling of Figure 1, which is the tiling of $D_{1}$ in dashed line. $D_{1}$ is:

$$
\begin{gathered}
D_{1}=\left\{\left(\xi_{1}, \xi_{2}\right) \in \hat{\mathbb{R}}^{2}:\left|\xi_{2}\right| \geq \frac{1}{8},\left|\frac{\xi_{1}}{\xi_{2}}\right| \leq 1\right\} \\
\text { Let } \boldsymbol{A}_{1}=\left(\begin{array}{ll}
2 & 0 \\
0 & 4
\end{array}\right), \boldsymbol{B}_{1}=\left(\begin{array}{ll}
1 & 0 \\
1 & 1
\end{array}\right), \text { and } \psi^{(1)} \text { is as follows: } \\
\hat{\psi}^{(1)}(\boldsymbol{\xi})=\hat{\psi}^{(1)}\left(\xi_{1}, \xi_{2}\right)=\hat{\psi}_{1}\left(\xi_{2}\right) \hat{\psi}_{2}\left(\frac{\xi_{1}}{\xi_{2}}\right)
\end{gathered}
$$

where $\hat{\psi}_{1}, \hat{\psi}_{2}$ is defined the same as the previous equation. Then, we get a Parseval frame for $L^{2}\left(D_{1}\right)$, that is as follows:

$\left\{\psi_{j, l, k}^{(1)}(\boldsymbol{x})=2^{\frac{3 j}{2}} \psi^{(1)}\left(\boldsymbol{B}_{1}^{l} \boldsymbol{A}_{1}^{j} \boldsymbol{x}-\boldsymbol{k}\right): j \geq 0,-2^{j} \leq l \leq 2^{j}-1, \boldsymbol{k} \in \mathbb{Z}^{2}\right\}$

If $f \in L^{2}\left(\mathbb{R}^{2}\right)$, then its continuous shearlet transform is defined as follows:

$$
\mathrm{SH}_{\psi}=\left\langle f, \psi_{j, l, k}^{(d)}\right\rangle
$$

where $j \geq 0, l=-2^{j} \sim 2^{j}-1, \boldsymbol{k} \in \mathbb{Z}^{2}, d=0,1$.

The discrete shearlet in [4] uses nonsubsampled Laplace pyramid to achieve multi-scale decomposition, which greatly increases the redundancy of the transform. Also, it greatly increases the computing time.

Due to the high redundancy and massive calculation of nonsampled wavelet, DTCWT, one that is constructed through a pair of wavelet trees, is proposed in [8]. Although DTCWT is an invertible quasi shift invariant and its 1-D case a redundancy of 2, the design of these quadrature wavelet pairs is so complicated that it can be done only through approximations. It means that the DTCWT requires special mother wavelet function. To overcome this restraint, in [7], Firoiu has proposed a new shift invariant called HWT using Hilbert transform and a two-stage mapping-based complex wavelet transform (MBCWT) in soft space [9]. And she also gives the proof that HWT is equivalent to DTCWT. That means that
HWT's redundancy ratio is 2, the same as that of DTCWT. Moreover, HWT can be realized through classical mother wavelet function like those conceived by Daubechies. Using this method, we can get a higher degree of shift invariance and a better directional selectivity [9].

So we use HWT to achieve multi-scale decomposition and apply shear filter bank to the HWT coefficients. For short, we name this new algorithm hyperanalytic shearlet transform, which holds the advantages of a simpler structure and a higher sparseness. What is more, it has greatly improved the redundancy compared with shearlet transform and greatly reduced the computing time. We first introduce HWT and then construct hyperanalytic shearlet.

Figure 3 gives the construction of 2-D HWT. We can see that $f^{D_{M}^{4}}$ denotes the low-pass coefficients and $z_{k}(k= \pm i$, $\pm r$ ) denotes the high-pass coefficients.

Figure 3 shows that the 2-D HWT of the image $f\left(x_{1}, x_{2}\right)$ can be computed with the aid of the 2-D DWT and its associated hypercomplex image. To simplify the calculation, the hypercomplex mother wavelet function associated to the real mother wavelet $\psi\left(x_{1}, x_{2}\right)$ is defined [7,9] as:

$$
\begin{aligned}
\psi_{a}\left(x_{1}, x_{2}\right)= & \psi\left(x_{1}, x_{2}\right)+i H_{x_{1}}\left\{\psi\left(x_{1}, x_{2}\right)\right\} \\
& +j H_{x_{2}}\left\{\psi\left(x_{1}, x_{2}\right)\right\}+k H_{x_{1}}\left\{H_{x_{2}}\left\{\psi\left(x_{1}, x_{2}\right)\right\}\right\}
\end{aligned}
$$

where $i^{2}=j^{2}=-k^{2}=-1$ and $i j=j i=k, H_{x_{1}}$ and $H_{x_{2}}$ respectively denotes the 1-D Hilbert transform of the lines and columns of the input image. So the 2-D HWT of the image $f\left(x_{1}, x_{2}\right)$ shown in Figure 3 is as follows:

$$
\begin{aligned}
\operatorname{HWT}\left\{f\left(x_{1}, x_{2}\right)\right\}= & \left\langle f\left(x_{1}, x_{2}\right), \psi_{a}\left(x_{1}, x_{2}\right)\right\rangle \\
= & \operatorname{DWT}\left\{f\left(x_{1}, x_{2}\right)\right\}+i \operatorname{DWT}\left\{H_{x_{1}}\left\{f\left(x_{1}, x_{2}\right)\right\}\right\} \\
& +j \operatorname{DWT}\left\{H_{x_{2}}\left\{f\left(x_{1}, x_{2}\right)\right\}\right\} \\
& +k \operatorname{DWT}\left\{H_{x_{1}}\left\{H_{x_{2}}\left\{f\left(x_{1}, x_{2}\right)\right\}\right\}\right\}
\end{aligned}
$$

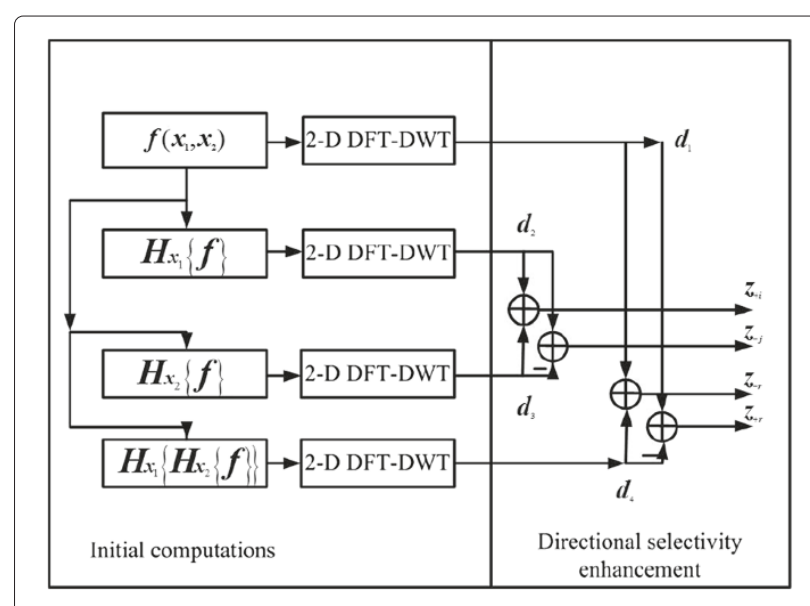

Figure 3 2-D HWT implementation architecture. 
Next, we will demonstrate the construction for hyperanalytic shearlet. Let the dilations $\boldsymbol{A}^{j}$ be associated with scale transformation, and the matrices $\boldsymbol{B}^{l}$ be associated to areapreserving geometrical transformation. For $\forall\left(\xi_{1}, \xi_{2}\right) \in$ $\hat{\mathbb{R}}^{2}, j \geq 0, \boldsymbol{k} \in \mathbb{Z}^{2}, d=0,1, l=-2^{j} \sim 2^{j}-1$, the hyperanalytic shearlet transform of $f \in L^{2}\left(\mathbb{R}^{2}\right)$ can be computed via:

$$
\left\langle f, \psi_{j, l, \boldsymbol{k}}^{(d)}\right\rangle=2^{\frac{3 j}{2}} \int \hat{f}(\boldsymbol{\xi}) \overline{V\left(2^{-2 j} \boldsymbol{\xi}\right) W_{j, l}^{(d)}} e^{2 \pi i \xi \boldsymbol{A}^{-j} \boldsymbol{B}^{-l} \boldsymbol{k}} d \boldsymbol{\xi}
$$

where $\psi$ is wavelet function of hyperanalytic shearlet, $W$ is a window function localized on a pair of trapezoid. And $V\left(2^{-2 j} \xi\right)$ is the Fourier coefficients of the multiscale analysis. The 2-D discrete Fourier transform (DFT) of image $\in L^{2}\left(\mathbb{R}^{2}\right)$ is $\hat{f}\left[k_{1}, k_{2}\right]\left(-\frac{N}{2} \leqslant k_{1}, k_{2} \leqslant \frac{N}{2}\right)$. Here, we adopt the convention that brackets [,] denote arrays of indices, parentheses (,) denote function evaluations, and $N \times N$ denotes the image size.

At the $j$ th scale, we utilize HWT instead of Laplace transform to compute the equation $\hat{f}\left(\xi_{1}, \xi_{2}\right)$ $\overline{V\left(2^{-2 j} \xi_{1}, 2^{-2 j} \xi_{2}\right)}[6,7,9]$. So we can decompose the father sub-band coefficients $f_{a}^{j-1}\left[n_{1}, n_{2}\right]$ into one low-pass subband coefficient $f_{a}^{j}\left[n_{1}, n_{2}\right]$ whose size is half of the father sub-band size (In fact, it produces two low-pass sub-band coefficients through HWT. In order to conduct a HWT pyramids conveniently, we make two low-pass sub-band coefficients decomposed from each scale to one low-pass sub-band coefficient.) and six high-pass sub-band coefficients $f_{d(\gamma)}^{j}\left[n_{1}, n_{2}\right], \gamma=0 \sim 6$. The sizes of $f_{a}^{j}\left[n_{1}, n_{2}\right]$ and $f_{d(\gamma)}^{j}\left[n_{1}, n_{2}\right]$ are $N_{j}^{a}=2^{-j+1} N$ and $N_{j}^{d}=2^{-j} N$, respectively. So we have

$$
\hat{f}_{d(\gamma)}^{j}\left[n_{1}, n_{2}\right]=\hat{f}\left(\xi_{1}, \xi_{2}\right) \overline{V\left(2^{-2 j} \xi_{1}, 2^{-2 j} \xi_{2}\right)}
$$

To achieve the direction filter of the high frequency coefficients after decomposition of $f_{d(\gamma)}^{j}\left[n_{1}, n_{2}\right]$, we should construct a shear direction filter using window function. Let $\hat{\delta}_{p}$ represent the DFT of the delta function in the pseudo-polar grid. And $\varphi_{p}$ is the mapping function from the Cartesian grid to the pseudo-polar one [10], which can be described as a selection matrix $S$ with the property that its elements $s_{i, j}$ satisfy the property $s_{i, j}^{2}=s_{i, j}$. Then, the hyperanalytic shearlet coefficients $\hat{f}_{d(\gamma)}^{j}\left[n_{1}, n_{2}\right] \hat{w}_{j, l}^{s}\left[n_{1}, n_{2}\right]$ in the Cartesian grid are as follows:

$$
\hat{w}_{j, l}^{s}\left[n_{1}, n_{2}\right]=\varphi_{p}^{-1}\left(\hat{\delta}_{p}\left[n_{1}, n_{2}\right] \tilde{W}\left[2^{j} n_{2}-l\right]\right)
$$

where $\tilde{W}$ is a Meyer window function. Finally, let $w_{j, l}^{s}$ denote inverse Fourier transform of $\hat{w}_{j, l}^{s}$. Then, we give the hyperanalytic shearlet transform of $f \in L^{2}\left(\mathbb{R}^{2}\right)$, which is defined as:

$$
\operatorname{DST}_{\psi}(f)=f_{a}^{J}+\sum_{j=0}^{J} \sum_{l=-2}^{2^{j}-1} \sum_{\gamma=0}^{3} f_{d(\gamma)}^{j} * w_{j, l}^{s}
$$

Here, $J$ is the final scale.

The hyperanalytic shearlet eliminates the aliasing phenomenon and has better direction selectivity and lower redundancy than the nonsubsampled shearlet. Moreover, hyperanalytic shearlet realizes the multi-scale decomposition by using addition, subtraction, and FFT. All the analysis shows that the calculation speed of hyperanalytic shearlet is quite fast; at the mean time, it can have a good visual effect as nonsubsampled shearlet does. Then, we apply it to separate an image into its morphologically different contents.

\section{Apply hyperanalytic shearlet transform to geometric separation}

It is an important technique to separate an image into its morphologically different contents using MCA [1] from prior information about the type of features to be decomposed. Recently, Donoho and Kutyniok presented a mathematical framework in [3] for image separation and constructed geometric separation mathematical theory of separating pointlike from curvelike features. And Kutyniok and Lim gave a new wavelet-shearlet dictionary based on their analysis in [4]. After analyzing the advantages of hyperanalytic shearlet in Section 2, it is natural to construct a new combined dictionary of wavelet and hyperanalytic shearlet. In order to improve the computational efficiency, we first apply the new dictionary to solve the problem about the separation of pointlike and curvelike features to an $l_{1}$ optimization model. And then, we use fast alternating direction method in [11] to obtain the optimal solution. Compared with separation algorithms using wavelet and shearlet in various ways, such approach is proved superior by numerical results especially when it comes to the speed. In the following, we briefly review this theoretical approach to the geometric separation problem and present our method.

Let function $P$ denote a 'pointlike' object and function $C$ denote a 'curvelike' object. The image function $f$ is expressed as:

$$
f=P+C
$$

The geometric separation problem now is turned into recovering $P$ and $C$ from the observed signal $f$. Since curvilinear singularities can be sparsely represented by shearlet, they can also be sparsely represented by hyperanalytic shearlet, while point singularities can be optimally sparsely represented by wavelet. So we choose the 
orthonormal separable Meyer wavelet and hyperanalytic shearlet to construct a dictionary to separate pointlike from curvelike structures.

Let $\left(F_{j}\right)_{j}$ denote a family of filters including wavelet and hyperanalytic shearlet filters. Then, the function $f$ can be decomposed into pieces $f_{j}$ with different scales $j$ based on different sub-band filters. So we can get the equation $f_{j}=$ $F_{j} * f$, and the original function can be reconstructed by using $f=\sum_{j} F_{j} * f_{j}, f \in L^{2}\left(\mathbb{R}^{2}\right)$.
With these conceptions above, we can solve the geometric separation problem step by step. For this, the model (15) can be rewritten as $f_{j}=P_{j}+C_{j}$ for every scale. Let $\Phi_{1}$ and $\Phi_{2}$ be the basis of orthonormal separable Meyer wavelet and hyperanalytic shearlet. Then, for each scale $j$, we consider the optimization problem as follows:

$$
\left(\hat{W}_{j}, \hat{S}_{j}\right)=\arg \min _{W_{j}, S_{j}}\left\|\Phi_{1}^{T} W_{j}\right\|_{1}+\left\|\Phi_{2}^{T} S_{j}\right\|_{1} \text { s.t. } f_{j}=W_{j}+S_{j}
$$

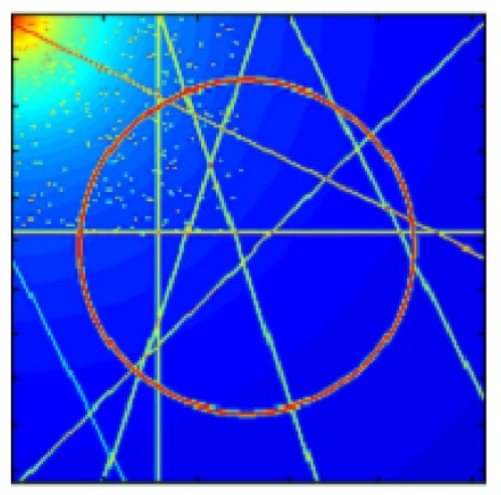

a

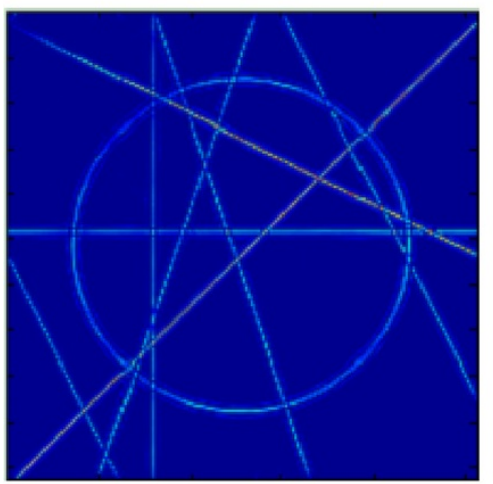

c

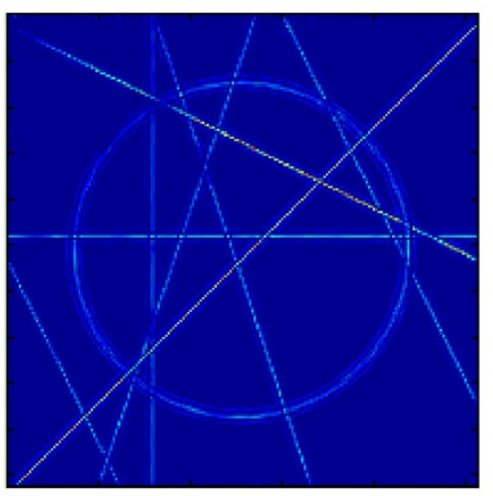

e

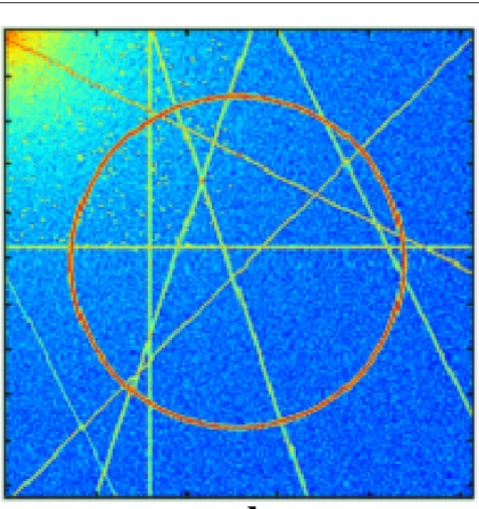

b

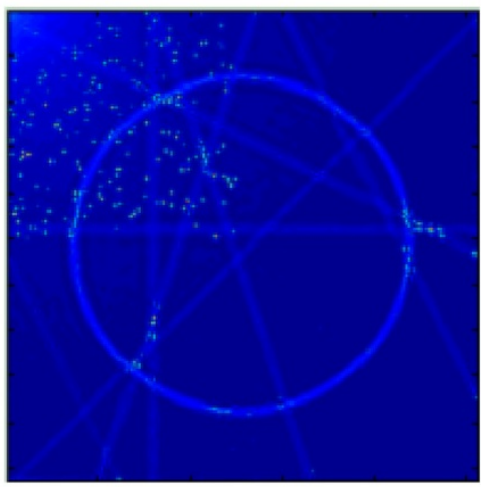

d

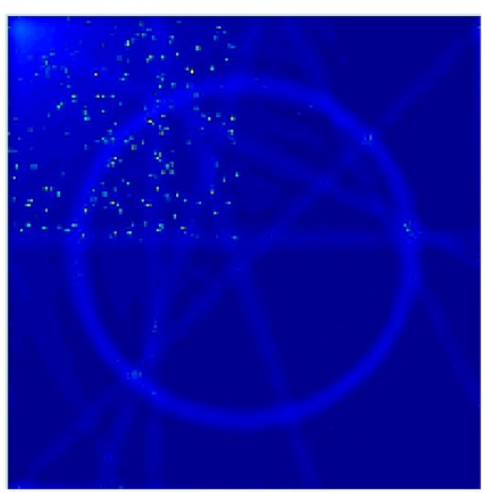

f

Figure 4 Comparison results between W-SD and W-HSD for artificial image, computing times: $164.75 \mathrm{~s}$ (W-SD) and $30.43 \mathrm{~s}$ (W-HSD). (a) Original image $(512 \times 512)$. (b) Noise image. (c) Curvelike component (W-SD). (d) Pointlike component (W-SD). (e) Curvelike component (W-HSD). (f) Pointlike component (W-HSD). 


\section{Table 1 The performance of different methods}

\begin{tabular}{lcc}
\hline The dictionary using for image separating & W-SD & W-HSD \\
\hline The computing times $(\mathrm{s})$ & 164.75 & 30.43 \\
The sep-degree & 7.0835 & 7.3758 \\
\hline
\end{tabular}

where $W_{j}$ denotes the pointlike signal composition of $f_{j}$, $S_{j}$ denotes the curvelike signal composition of $f_{j} . \Phi_{1}^{T} W_{j}$ denotes the Wavelet coefficients of the signal $W_{j}$, and $\Phi_{2}^{T} S_{j}$ denotes hyperanalytic shearlet coefficients of $S_{j}$.
Obviously, the solution of (16) is the $l_{1}$ minimization problem. In practice, the signal $f$ is often contaminated by noise, and in [4], the authors have presented a new model to solve this problem adaptively. That is, for each scale $j$, the optimization problem can be presented through the following model:

$$
\left(\hat{W}_{j}, \hat{S}_{j}\right)=\arg \min _{W_{j}, S_{j}}\left\|\Phi_{1}^{T} W_{j}\right\|_{1}+\left\|\Phi_{2}^{T} S_{j}\right\|_{1}+\lambda\left\|f_{j}-W_{j}-S_{j}\right\|_{2}^{2}
$$

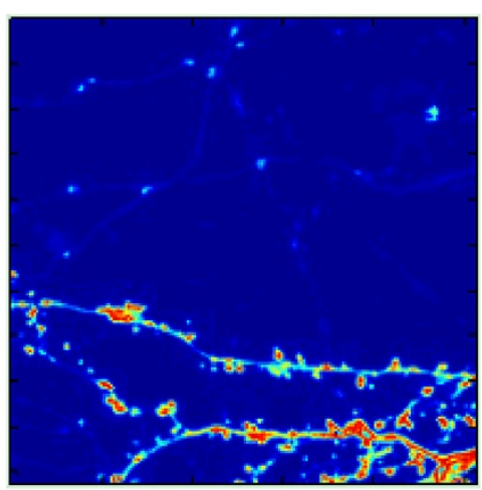

a

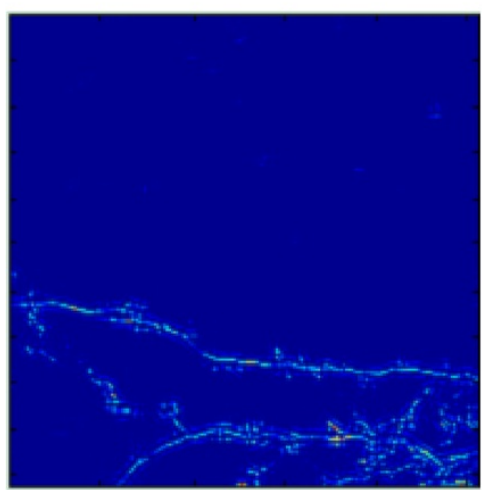

c

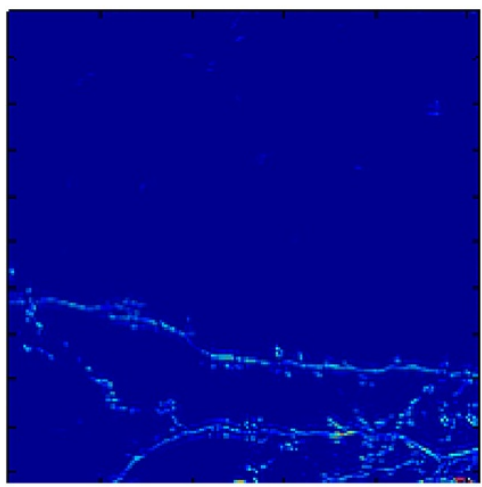

e

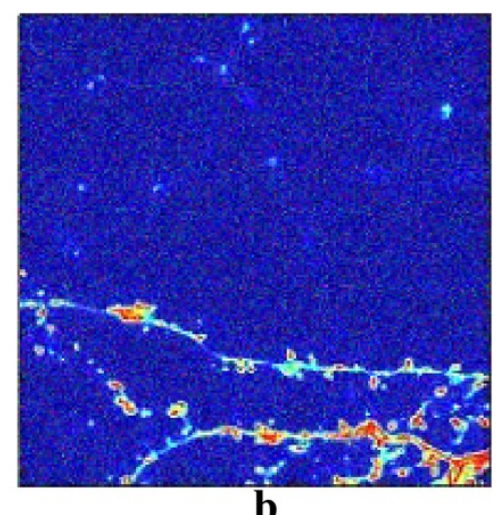

b

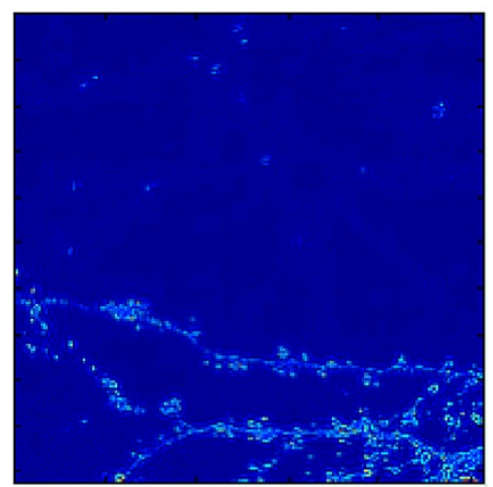

d

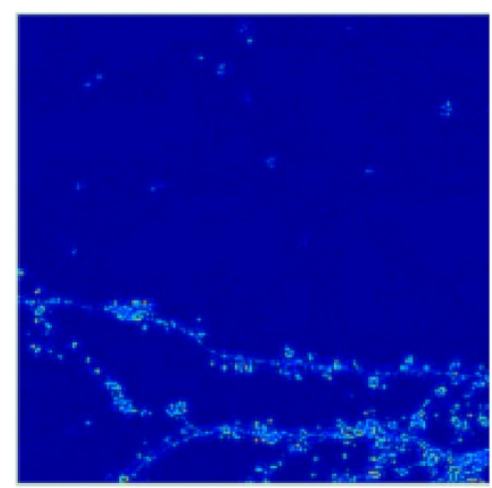

f

Figure 5 Comparison results between W-SD and W-HSD for biological image, computing times: 167.77 s (W-SD) and $31.23 \mathrm{~s}$ (W-HSD). (a) Original image $(512 \times 512)$. (b) Noise image $(512 \times 512)$. (c) Curvelike component (W-SD). (d) Pointlike component (W-SD). (e) Curvelike component (W-HSD). (f) Pointlike component (W-HSD). 
In (17), the noise cannot be represented sparsely by either wavelet or hyperanalytic hearlet, and then it can be related with the residual $f_{j}-W_{j}-S_{j}$.

It costs a lot of computational time to solve (17) for all scales. Kutyniok and Lim [4] show that it has been sufficient for separating pointlike from curvelike structures with sufficiently large scales $j$. So we re-weight the different scales coefficients which strengthen the highfrequency sub-band to avoid high complexity like [4]. For each scale $j$, we choose such a weight vector $\boldsymbol{w}$, and the elements of $\boldsymbol{w}$ are all positive and $w_{j}<w_{i}$, if $j<i$. Then, we can get the new signal $\tilde{f}$ by computing

$$
\tilde{f}=\sum_{j} w_{j}\left(F_{j} * f_{j}\right), f \in L^{2}\left(\mathbb{R}^{2}\right)
$$

where $f=\sum_{j} F_{j} * f_{j}$ and $f_{j}=F_{j} * f$.

From (17) and (18), we can separate pointlike from curvelike structures by solving the follow problem:

$$
(\hat{W}, \hat{S})=\arg \min _{W, S}\left\|\Phi_{1}^{T} W\right\|_{1}+\left\|\Phi_{2}^{T} S\right\|_{1}+\lambda\|\tilde{f}-W-S\|_{2}^{2}
$$

We can focus on the high frequencies by using signal $\tilde{f}$ which greatly improves the computational efficiency. But we do not calculate the optimization problem like in [4] because BCR method in [12] is not precise enough and also it is slow. In this paper, we use the fast numerical schemes - FADM in [11] - to solve (19) since we can get more accurate results and have a faster computing speed. Apply FADM to (19).

(a) First, fix $S$; then, we can get the solution of $W$ :

$$
\begin{aligned}
\hat{W}^{(k)} & =\underset{W}{\arg \min }\left\|\Phi_{1}^{T} \hat{W}^{(k-1)}\right\|_{1}+\left\|\Phi_{2}^{T} S\right\|_{1}+\lambda_{2}\left\|\tilde{f}-\hat{W}^{(k-1)}-S\right\|_{2}^{2} \\
& =\underset{W}{\arg \min }\left\|\Phi_{1}^{T} \hat{W}^{(k-1)}\right\|_{1}+\lambda_{2}\left\|g_{1}^{(k)}-\hat{W}^{(k-1)}\right\|_{2}^{2}
\end{aligned}
$$

(b) Then, fix $W$; then, we can get the solution of $S$ :

$$
\begin{aligned}
\hat{S}^{(k)} & =\underset{S}{\arg \min }\left\|\Phi_{1}^{T} W\right\|_{1}+\left\|\Phi_{2}^{T} \hat{S}^{(k-1)}\right\|_{1}+\lambda_{2}\left\|\tilde{f}-\hat{S}^{(k-1)}-W\right\|_{2}^{2} \\
& =\underset{S}{\arg \min }\left\|\Phi_{1}^{T} \hat{S}^{(k-1)}\right\|_{1}+\lambda_{1}\left\|g_{2}^{(k)}-\hat{S}^{(k-1)}\right\|_{2}^{2}
\end{aligned}
$$

$$
\text { where } k \geq 1, g_{1}^{(k)}=\tilde{f}-\hat{S}^{(k-1)}, g_{2}^{(k)}=\tilde{f}-\hat{W}^{(k)} \text {. }
$$

Minimization problem (20) and (21) can be uniformly written as the following minimization problem:

$$
\hat{v}=\underset{v \in \mathbb{R}^{N}}{\arg \min } \rho(v)+\lambda\|g-v\|_{2}^{2}
$$

where $\rho(\cdot)$ denotes $l_{1}$ norm. Then, we can solve (22) by using two-step iterative shrinkage algorithm in [13], which is an easy and fast method, see the details in [13].

In order to verify the reliability and validity of the proposed algorithm, we introduce a measure named sepdegree to evaluate the separation performance of our transform. $f$ denotes the noise image, $f_{c}$ denotes the separated image containing curvelike features, and $f_{p}$ denotes the separated image containing pointlike features. Let $\mu(f)$ denote the mathematical expectation of $f$. Then, we can define the sep-degree as follows:

Definition 1. The sep-degree $\zeta$ of an algorithm for separating pointlike and curvelike features can be defined as the following:

$$
\begin{aligned}
& \varpi=\frac{\sum_{i=1}^{M} \sum_{j=1}^{N}\left(f^{G}\right)_{i, j} \cdot\left(\left(f^{S}\right)^{G}\right)_{i, j}}{\sqrt{\left(\sum_{i=1}^{M} \sum_{j=1}^{N}\left(\left(f^{G}\right)_{i, j}\right)^{2}\right) \cdot\left(\sum_{i=1}^{M} \sum_{j=1}^{N}\left(\left(\left(f^{S}\right)^{G}\right)_{i, j}\right)^{2}\right)}} \\
& \varsigma=\frac{\varpi}{\operatorname{abs}\left(\mu\left(f-f^{S}\right)\right)}
\end{aligned}
$$

where $(f)^{G}=\operatorname{Gradient}(f)$, which denotes the gradient map of the image $f$, and $f^{S}=f_{p}+f_{c},\left(f^{S}\right)^{G}=\operatorname{Gradient}\left(f^{S}\right)$. $a b s(\cdot)$ denotes the absolute value of the function, and $\varpi$ denotes the similarity between $(f)^{G}$ and $\left(f^{S}\right)^{G}$.

The definition of sep-degree shows that the separation method's performance is better when $\varsigma$ is larger. It is because that $\mu\left(f-f^{S}\right)$ should be smaller enough for a better separating method, which means we get a most approximate image of $f$. From Jensen's inequality, we know $\varpi \leq 1$ in (23), and the closer $\varpi$ to 1 is, the more similar $(f)^{\bar{G}}$ and $\left(f^{S}\right)^{G}$ are, which means that the extracted curvelike component is more perfect. For a better separation method, $\mu\left(f-f^{S}\right)$ should be smaller and $\varpi$ should be larger, so sep-degree $\zeta$ should be larger based on (24). In the following, $\zeta$ is used to distinguish the numerical experiments.

\section{The numerical results}

In this section, we compare our scheme of wavelet and hyperanalytic shearlet dictionary (W-HSD) with FADM against the separation algorithm based on wavelet and

Table 2 The performance of different methods apply to neuron image

\begin{tabular}{lcc}
\hline The dictionary using for image separating & W-SD & W-HSD \\
\hline The computing times (s) & 167.77 & 31.23 \\
The sep-degree & 7.1825 & 7.6578
\end{tabular}


shearlet dictionary (W-SD) with BCR in [4]. And the scale of all is four; for hyperanalytic shearlet and nonsubsampled shearlet, the direction vector is [ [ $\left.\begin{array}{lll}2 & 3 & 4\end{array}\right]$. All routines were run using the Matlab R2009a which is based on an Intel 2.00GHz CPU.

The different methods for separating pointlike features and curvelike features from an artificial image are shown in Figure 4, and Table 1 shows the computing times and sep-degree of separating methods through W-SD and WHSD.

In the experimental results, Figure $4 \mathrm{a}$ is an artificial image consisting of a composition of pointlike and curvelike structures on a smooth background, and Figure $4 \mathrm{~b}$ is the same image added by some additive white Gaussian noise with variance 20 . Figure $4 \mathrm{c}$ shows the curvelike component of image separating by W-SD, and Figure $4 \mathrm{~d}$ shows the pointlike component of image separating by W-SD. Correspondingly, Figure 4e,f shows the curvelike and pointlike components of image separating by W-HSD. Compared with Figure 4c separated via W-SD method, Figure 4e shows that W-HSD does not keep all circle-like contents, which makes the circle of our scheme a little pale. Though the visual effect of Figure 4e is just a little worse than Figure 4c, the visual effect of Figure $4 \mathrm{f}$ is much better than Figure 4d. From Figure 4d, we know that the separating method W-SD can bring some visible artifacts, but our new algorithm will solve this problem. Finally, the comparison of the computing time shows that the separation can be much faster performed by our scheme because hyperanalytic shearlet has a low redundancy which greatly reduces the computational complexity. Table 1 shows that our algorithm can reduce the computing time to one fifth of the time of separating image by W-SD. Table 1 also shows that our algorithm can get a higher sep-degree than separating image by W-SD. It means that our algorithm can get a better performance in separating an artificial image consisting of a composition of pointlike and curvelike structures.

Let us consider the performance of our scheme and W-SD on the real-world images. Figure 5 a shows the experimental results on a test image of a neuron generated by fluorescence microscopy from the research group by Roland Brandt, which is composed of 'spines' (pointlike features) and 'dendrites' (curvelike features). Figure 5b is the noise image added by some additive white Gaussian noise. Comparing Figure $5 \mathrm{c}$ with Figure 5e, we see that our scheme extracts the curvelike structures much more precise than W-SD. Comparing Figure 5d with Figure 5f, we can also see that our scheme extracts the pointlike structures much more clearly than W-SD, and the computation time is greatly reduced, also shown in Table 2 .

Table 2 shows that our algorithm gets a better performance in separating a neuron image of a composition of pointlike and curvelike structures.

\section{Conclusions}

This paper proposes a novel approach of separation of pointlike and curvelike features exploiting a new combined dictionary of wavelet and hyperanalytic shearlet and defines a new objective measure called sep-degree to evaluate the separation performance. The experimental results demonstrate that the proposed method is more applicable to geometric separation. It has a better visual effect as wavelet and shearlet dictionary, and its computing time decreases sharply.

\section{Competing interests}

The authors declare that they have no competing interests.

Received: 28 February 2014 Accepted: 21 April 2014

Published: 9 May 2014

\section{References}

1. JL Starck, M Elad, DL Donoho, Image decomposition via the combination of sparse representation and a variational approach. IEEE Trans. Image Process. 14(2), 1570-1582 (2005)

2. DL Donoho, G Kutyniok, Microlocal analysis of the geometric separation problems. Comm. Pure Appl. Math. 66(1), 1-47 (2013)

3. DL Donoho, G Kutyniok, Geometric separation using a wavelet-shearlet dictionary, in SampTA'09, Marseille, France (IEEE, 2009), pp. 1-5

4. G Kutyniok, WQ Lim, Image separation using wavelets and shearlets, in Curves and Surfaces, Avignon, France (Springer, Heidelberg, 2010), pp. 416-430

5. WQ Lim, The discrete shearlets transform: a new directional transform and compactly supported shearlets frames. IEEE Trans. Image Process. 19(5), 1166-1180 (2010)

6. G Easley, D Labate, WQ Lim, Sparse directional image representation using the discrete shearlets transform. Appl. Comput. Harmonic Anal. 25(1), 25-46 (2008)

7. I Adam, C Nafornita, JM Boucher, A Isar, A new implementation of the hyperanalytic wavelet transform, in ISSCS 2007, lasi, Romani (IEEE, 2007), pp. 401-404

8. NG Kingsbury, Shift invariant properties of the dual-tree complex wavelet transform. IEEE Comput. Soc. 47(3), 1221-1224 (1999)

9. I Firoiu, C Nafornita, JM Boucher, A Isar, Image denoising using a new implementation of the hyperanalytic wavelet transform. IEEE Trans. Instrum. Meas. 58(8), 2410-2416 (2009)

10. F Colonna, GR Easley, Generalized discrete radon transforms and their use in the ridgelet transform. J. Math. Imag. Vis. 23(2), 145-165 (2005)

11. M Tao, Fast alternating direction method of multipliers for total-variation-based image restoration. J. Southeast Univ. 27(4), 379-383 (2011)

12. S Sardy, AG Bruce, P Tseng, Block coordinate relaxation methods for nonparametric wavelet denoising. J. Comput. Graph. Stat. 9(2), 361-379 (2000)

13. J Bioucas-Dias, M Figueiredo, A new twist: two-step iterative shrinkage/thresholding algorithms for image restoration. 16(12), 2992-3004 (2007)

doi:10.1186/1687-6180-2014-63

Cite this article as: Liu et al:: Apply hyperanalytic shearlet transform to geometric separation. EURASIP Journal on Advances in Signal Processing 2014 2014:63. 